\title{
GMR
}

\section{Expressed sequence tag analysis of functional genes associated with adventitious rooting in Liriodendron hybrids}

\author{
Nanchang, China \\ *These authors contributed equally to this study. \\ Corresponding author: F.X. Yu \\ E-mail: fxyu2000@126.com
}

Y.D. Zhong*, X.Y. Sun*, E.Y. Liu, Y.Q. Li, Z. Gao and F.X. Yu

The Key Laboratory of Horticultural Plant Genetic and Improvement of Jiangxi, Institute of Biology and Resources, Jiangxi Academy of Sciences,

Genet. Mol. Res. 15 (2): gmr.15027606

Received September 9, 2015

Accepted January 22, 2016

Published June 24, 2016

DOI http://dx.doi.org/10.4238/gmr.15027606

\begin{abstract}
Liriodendron hybrids (Liriodendron chinense $\mathrm{x} L$. tulipifera) are important landscaping and afforestation hardwood trees. To date, little genomic research on adventitious rooting has been reported in these hybrids, as well as in the genus Liriodendron. In the present study, we used adventitious roots to construct the first cDNA library for Liriodendron hybrids. A total of 5176 expressed sequence tags (ESTs) were generated and clustered into 2921 unigenes. Among these unigenes, 2547 had significant homology to the non-redundant protein database representing a wide variety of putative functions. Homologs of these genes regulated many aspects of adventitious rooting, including those for auxin signal transduction and root hair development. Results of quantitative real-time polymerase chain reaction showed that $A U X 1, I R E$, and $F B 1$ were highly expressed in adventitious roots and the expression of $A U X 1, A R F 1, N A C 1, R H D 1$, and IRE increased during the development of adventitious roots. Additionally, 181 simple sequence repeats were identified from 166 ESTs and more than $91.16 \%$
\end{abstract}


of these were dinucleotide and trinucleotide repeats. To the best of our knowledge, the present study reports the identification of the genes associated with adventitious rooting in the genus Liriodendron for the first time and provides a valuable resource for future genomic studies. Expression analysis of selected genes could allow us to identify regulatory genes that may be essential for adventitious rooting.

Key words: Liriodendron hybrids; cDNA library; Functional genes; Adventitious rooting; EST-SSRs

\section{INTRODUCTION}

The genus Liriodendron contains only two species: Liriodendron tulipifera, which is widely distributed in eastern North America, and Liriodendron chinense (Hemsl). Sarg, which is native to northern Vietnam and southern China (Parks and Wendel, 1990). Liriodendron hybrids, derived from sexual hybridization between $L$. chinense and $L$. tulipifera, are fast-growing hardwood trees and are widely used in fast-wood plantation construction, phytoremediation, and bioenergy industry. They are highly heterotic in many aspects, especially in having more attractive flowers, faster growth, and improved resistance to biotic and abiotic stresses (Parks et al., 1983). However, this economically valuable timber and landscaping tree is hard to breed due to difficulties in both sexual and clonal propagation.

Adventitious roots arise naturally upon adaptation to environmental stresses or from the aerial organs of plants. The formation of adventitious roots can be induced by indirect callus tissue formed because of mechanical damages. The ability to form adventitious roots can lead to rapid amplification of millions of cuttings from elite clones used for plantations. This capability is a heritable quantitative trait affected by many endogenous and environmental factors ( $\mathrm{Li}$ et al., 2009). Two major auxin response protein families, the $A U X / I A A$ (auxin/indole3 -acetic acid) and $A R F$ (auxin-response factor) gene families, involved in the regulation of adventitious rooting have been identified (Gutierrez et al., 2009). ARF6 and ARF8 positively regulate adventitious root initiation in Arabidopsis whereas ARF17 regulates it negatively (Gutierrez et al., 2009). The F-box protein TIR1 (transport inhibitor response 1) is an auxin receptor (Navarro et al., 2006), which regulates the degradation of Aux/IAA proteins and the consequent changes in the expression of auxin-regulated genes (Dharmasiri et al., 2005). The auxin receptor $N A C l$, acts downstream of TIRI and participates in lateral root stimulation (Xie et al., 2000; Guo et al., 2005). Auxin biosynthesis occurs mainly in young leaves, shoot and root apices, and auxin is redistributed by polar transport (Shimizu-Sato et al., 2009). The mutation of $A U X 1$, which encodes an auxin uptake carrier, results in reduced lateral root formation in Arabidopsis (Bennett et al., 1996). In the present decade, similar mechanisms of regulation of the adventitious root development have been reported in different organs of Arabidopsis (Gutierrez et al., 2012). However, due to the complex structure and intricate regulation of root systems, only a few genes involved in the adventitious rooting regulation have been identified (Ramírez-Carvajal et al., 2009), and the mechanism of adventitious root development in trees remains unclear.

Expressed sequence tags (ESTs) are valuable resources for gene discovery, gene mapping, and quantitative trait analysis. They can also be a good source of simple sequence 
repeats (SSRs), which are useful molecular markers in plant genetics and evolutionary studies. Recently, a large number of ESTs have been generated for many trees. Currently, there are three available EST databases (Albert et al., 2005; Liang et al., 2008; Jin et al., 2011; Liang et al., 2011) and one BAC library (Liang et al., 2007) for L. tulipifera and one EST database for L. chinense (Yang et al., 2014). Most of the cDNA libraries have been constructed from flowers, xylems, and leaves. The genomic resources for the hybrid yellow poplar are still limited, especially in terms of adventitious root development. Moreover, few DNA sequences are available in the NCBI database for Liriodendron hybrids with the exception of 232 miRNAs associated with somatic embryogenesis (Li et al., 2012). Considering the economic and ecological value of Liriodendron, the availability of transcriptome data of hybrid yellow poplar will contribute to Liriodendron breeding and related genetic research.

In the present study, we characterized the adventitious root transcriptomes of hybrid yellow poplar using the normalized cDNA library. Specifically, we aimed to identify the transcripts involved in adventitious rooting. The gene-based SSRs identified in the unigene assemblies can be applied to gene mapping and function analysis in the genus Liriodendron. To the best of our knowledge, this is the first report on the adventitious root transcriptome of Liriodendron hybrids, and the findings of this study could make significant contributions in improving the rooting of recalcitrant genotypes and in functional genomics studies of Liriodendron.

\section{MATERIAL AND METHODS}

\section{Plant materials}

Adventitious roots of hybrid yellow poplar (L. chinense $\mathrm{x}$ L. tulipifera) were derived from one ramet of clone 210. All the hardwood cuttings were obtained from an annual twig and dipped in a solution containing $300 \mathrm{ppm}$ indole-3-acetic acid (IAA) and $300 \mathrm{ppm}$ 3 -indolebutyric acid (IBA) for $15 \mathrm{~s}$. The trees were kept in a hotbed at $25^{\circ}-30^{\circ} \mathrm{C}$ during the day and rooted after 25-40 days. The process of adventitious rooting in hybrid yellow poplar cuttings was performed as described previously (Zhang and Fang, 2003). Tissues of three independent biological replicates were collected at four different phases (Figure 1): I) proliferation of vascular cambium cells at the base of the cuttings (callus was translucent at the early stage); II) dedifferentiation of vascular cambium cells and formation of root initials (callus looked milky white); III) growth and development of root primordium (adventitious roots elongated by approximately $1 \mathrm{~cm}$ ); IV) emergence of adventitious roots from the lentice or callus of the cuttings (adventitious roots elongated by more than $2 \mathrm{~cm}$ ). All the harvested samples were washed with $70 \%$ ethanol, immediately frozen in liquid nitrogen, and stored at $-80^{\circ} \mathrm{C}$, until RNA extraction.

\section{RNA extraction, library construction, and EST generation}

Total RNA was extracted from different stages of adventitious roots according to a modified Trizol (Invitrogen, Shanghai, China) method and was purified using RNeasy Plant Mini Kit (Qiagen, Hilden, Germany). RNA was dissolved in RNase-free (diethylpyrocarbonatetreated) water. The isolation and purification of mRNA was conducted using FastTrack ${ }^{\circledR} 2.0$ 
Kit (Invitrogen, Shanghai, China) according to the manufacturer protocol. An uncut cDNA library was constructed using CloneMiner (Invitrogen) according to the manufacturer instructions. The first strand of cDNA was synthesized by priming poly(A) RNA with BiotinattB2-Oligo(dT) primer [5'-Biotin-GGCGGCCGCACAACTTTGTACAAGAAAGTTGGGT $\left.(\mathrm{T})_{19} \mathrm{D}-3^{\prime}\right]$ using SuperScript ${ }^{\mathrm{TM}}$ II reverse transcriptase (Invitrogen), and the second strand was synthesized using the first cDNA strand as a template by E. coli DNA polymerase(TaKaRa, Dalian, China). An attB1 adapter was ligated to the 5'-end of the cDNA, while biotin prevented ligation of the attB1 adapter (5'-TCGTCGGGGACAACTTTGTACAAAAAAGTTGG-3', and 3'-CCCCTGTTGAAACATGTTTTTTCAACCp-5') to the 3'-end of the cDNA that had been selected and blunt ended. Thereafter, GatewayR technology, which constituted two recombination reactions including the BP reaction and LR reaction in the cDNA library construction kit, was used. The library constructed in pDONE ${ }^{\mathrm{TM}} 222$ (Invitrogen) was amplified in ElectroMAX ${ }^{\mathrm{TM}}$ DH10B ${ }^{\mathrm{TM}} \mathrm{T} 1$ Phage-Resistant cells. Finally, the cDNA library titer was calculated by performing the plating assay for an insert size greater than $500 \mathrm{bp}$.
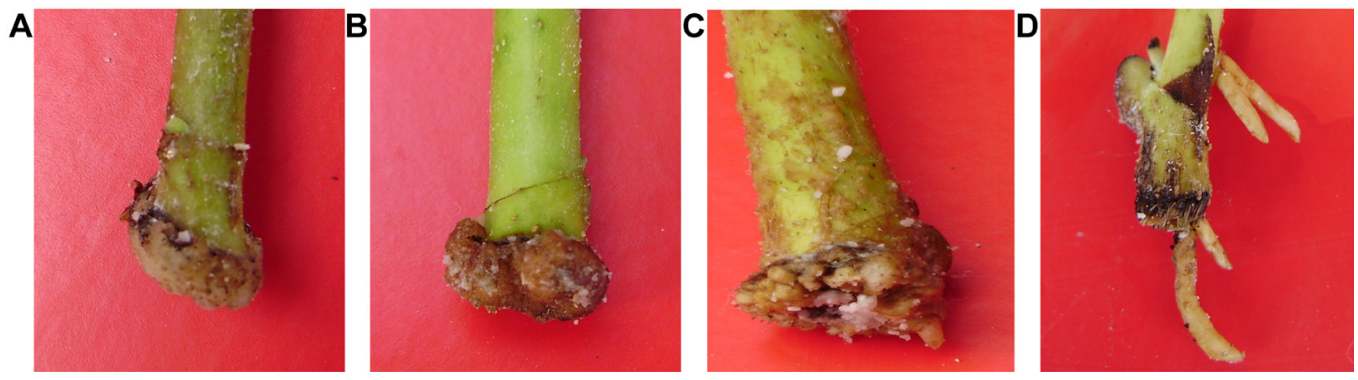

Figure 1. Four developmental phases during adventitious rooting of Liriodendron hybrids. A. proliferation of vascular cambium cells at the base of the cuttings; B. dedifferentiation of vascular cambium cells and formation of a root initial; C. growth and development of root primordium; D. emergence of adventitious roots from the lentice or the callus of the cuttings.

The cDNA library was derived from the uncut cDNA library by saturation hybridization with genomic DNA of hybrid yellow poplar on magnetic beads. Firstly, the genomic DNA was extracted using an Axygen genomic DNA miniprep kit (Axygen Biosciences, USA). DNA was incubated with HindIII, purified, and ligated with Biotin-dUTP. Then, the DNA was incubated with BamHI and purified. The immobilized affinity system was constructed by adsorbing the magnetic beads using the DYNAL M280 magnetic bead kit (Invitrogen). The driver DNA of the immobilized affinity system was pre-hybridization at $65^{\circ} \mathrm{C}$ for $1 \mathrm{~h}$ in a hybridization oven (Hb-1000, UVP, Upland, CA, USA). Thereafter, single-stranded plasmids of the denatured cDNA library were hybridized with the driver DNA in the presence of $0.5 \%$ SDS (w/v), 6X SSC, and 5X Denhardt's solution (Invitrogen). The reaction was performed for $16 \mathrm{~h}$ at $65^{\circ} \mathrm{C}$ to achieve a calculated $C_{0} t$ value of $5 \mathrm{~s}-\mathrm{M}$. The hybridization mixture was washed four times with washing buffer (1\% SDS (w/v) and 2X SSC) and separated from the magnetic beads using a magnetic separation rack (Invitrogen) by incubating at $75^{\circ} \mathrm{C}$ for 10 min before being renatured. The plasmids eluted from the magnet were concentrated by butanol extraction and ethanol precipitation. The recombined plasmid DNA was transformed into ElectroMAX ${ }^{\mathrm{TM}}$ DH10B ${ }^{\mathrm{TM}}$ T1 Phage Resistant cells by electroporation using the BTX 
ECM 630 electroporator (Genetronics, San Diego, CA, USA). The plasmid DNA prepared from these bacteria represents the amplified normalized cDNA library. The titer of this library was determined by XGal/IPTG screening. ESTs were generated by sequencing the cDNA library and approximately 5252 clones were randomly picked by Sanger sequencing.

\section{Data processing, assembly, and annotation}

All the sequences were trimmed of low quality segments at the 5'- and 3 '-ends by Phred score 20 prior to assembling the ESTs and then the vector and adaptor sequences were removed by Cross-match. Sequences longer than $100 \mathrm{bp}$ were clustered into unigenes, including the contigs and singletons using Phrap. Unigenes were imported and compared against the NCBI database using BLASTX and TBLASTX (minimum match identity $\geq 25 \%$ and $\mathrm{E}$ value $\leq 1 \mathrm{E}^{-10}$ ). The NCBI non-redundant nucleotide and protein databases were used for general annotation. The conserved domain information with potential functions of gene and gene ontology were predicted using Gene Ontology (GO; http://www.geneontology.org).

\section{Gene expression analysis}

Seven ESTs (Table 1) for adventitious rooting, including one gene for auxin transport $(A U X 1)$, three genes for auxin signal response $(A R F 1, N A C 1, F B 1)$, and three genes for root hair development (RHD1,RHL1,IRE), were selected for the study. Using the partial sequences obtained from the EST sequencing, we designed primers for detecting the expression patterns of these genes.

Total RNA extraction from tissues in four developmental phases of adventitious rooting (Figure 1) and various organs (adventitious roots, stems, and leaves) was performed as described above. One microgram DNase treated RNA was then reverse transcribed into cDNA using SuperScript III First-Strand Synthesis System (Invitrogen) with random primers. For quantitative real-time polymerase chain reaction (RT-PCR), specific primers (Tm, $58^{\circ}-61^{\circ} \mathrm{C}$ ) were designed to amplify a PCR segment of 50-150 bp using Primer Express Software version 3.0 (Applied Biosystems, Foster City, CA, USA) and Oligo6.6 (Molecular Biology Insights, Cascade, CO) (Table 1). Real-time PCR was conducted using the ABI 7500 Real-time PCR system (Applied Biosystems) and SYBR Green Realtime PCR Master Mix (Toyobo, Co., Ltd., Osaka, Japan) according to the manufacturer protocol. For each sample, the reactions were performed in a final volume of $20 \mu \mathrm{L}$ containing $10 \mu \mathrm{L} 2 \mathrm{X}$ SYBR Green Realtime PCR Master Mix, $1 \mu \mathrm{L}(10 \mu \mathrm{M})$ each primer, $3 \mu \mathrm{L}$ cDNA, and $5 \mu \mathrm{L}$ Milli-Q $\mathrm{H}_{2} \mathrm{O}$; three technical replicates were taken for each sample.

Table 1. Primers and PCR product size of the genes used for RT-PCR analysis.

\begin{tabular}{l|l|l|l|c}
\hline Gene name & Unigene number & Forward primer & Reverse primer & Product size (bp) \\
\hline$A U X 1$ & Contig89 & AAGGAAGCCGAGAAGGTTAG & CCGTTCAAGAGATCCATCA & 224 \\
\hline ARF1 & E11-K04302389-K2389 & GCTCTCATCCTCCCTTGT & CCTCCGTTCAAGAAGCC & 108 \\
\hline NAC1 & Contig669 & AGATGTTGGGTGGTGGAG & TGGCATCAGTGGCAGAG & 120 \\
\hline$R H D 1$ & Contig1663 & GCCACGGAAGGTCAAG & GGAAGCGGAGGTAGGAAG & 131 \\
\hline$I R E$ & Contig535 & TCCTCTCCGCCGTTGATT & GCCATCATAGCCCTTCTTCC & 218 \\
\hline$R H L 1$ & H11-K04304600-K4600 & AGAAGGGCCAGAGGAAGAA & TGCGGGAAATCAATGTAGAG & 130 \\
\hline$F B 1$ & C03-K04304147-K4147 & GGTCCGCAACAAGATACAA & ATCTGTCCCAACATCCCTAA & 122 \\
\hline Histone H3 & Contig924 & TCCACAGGAGGAAAGGCTC & GTGCAACAGTTCCAGGGCG & 115 \\
\hline
\end{tabular}


The amplification was conducted with 40 cycles of denaturation at $95^{\circ} \mathrm{C}$ for $15 \mathrm{~s}$, annealing and extension at $60^{\circ} \mathrm{C}$ for $1 \mathrm{~min}$, along with initial denaturation at $95^{\circ} \mathrm{C}$ for $1 \mathrm{~min}$. The histone $\mathrm{H} 3$ gene was used as an internal control. Data from the quantitative RT-PCR analysis for gene expression were transformed using the $2^{-\Delta \Delta \mathrm{Ct}}$ method.

\section{SSR development from ESTs}

SSRs were mined from the non-redundant ESTs by Simple Sequence Repeat Identification Tool (SSRIT) database (http://www.grameme.org/db/searches/ssrtool). The minimum number of repeats was nine for di-nucleotide repeats, six for tri-nucleotide, five for tetra-nucleotide, and four for penta- and hexa-nucleotide repeats. SSR primer pairs were designed from the flanking sequences, using the PRIMER3 software (www.vgenome.wi.mit. edu/cgibin/primer/primer3_www.cgi).

\section{RESULTS AND DISCUSSION}

\section{Library construction and characterization}

An uncut cDNA library was constructed from four developmental phases of adventitious roots of hybrid yellow poplar. The titer of this library consisted of $7.34 \times 10^{6}$ clones with more than $95 \%$ recombinant clones and the titer of the library was $3.72 \times 10^{5}$ with more than $95 \%$ recombinant clones after normalization. Four widely distributed housekeeping genes were then selected and specific primers for real time polymerase chain reaction (RT-

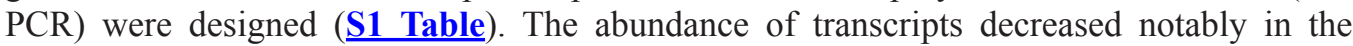
normalized cDNA library compared to the uncut samples. These results suggested that the normalized cDNA library had been established successfully, which facilitated further studies to identify the expressed genes in the developmental phases of adventitious rooting in hybrid yellow poplar.

In the present study, a normalized cDNA library was constructed from the developing adventitious roots of hybrid yellow poplar. The normalization efficiency was identified using the four housekeeping genes. There was a 35- to 85-fold reduction in abundance after the normalization. This normalization would greatly facilitate the enrichment of the library for rare genes, reduce the cost of sequencing, and increase the recovery rate of unigenes by avoiding redundant clones.

\section{Library sequencing and contig assembly}

A total of 5176 high quality ESTs were obtained after screening for vector sequences and excluding low quality sequences shorter than $100 \mathrm{bp}$ (Table 2). The read length of the ESTs ranged from 59 to $2349 \mathrm{bp}$, with an average length of $1001 \mathrm{bp}$, which was longer than floral bud ESTs of L. tulipifera, and petal and leaf ESTs of L. chinense (Liang et al., 2008; Liang et al., 2011; Yang et al., 2014) but was smaller than the secondary xylem ESTs of L. tulipifera (Jin et al., 2011). The GC content of 46.74\% was comparable to that of other transcriptomes in Liriodendron (Liang et al., 2008; Yang et al., 2014), but was different from those of Arabidopis (42.7\%) and rice (51.1\%; Kuhl et al., 2004). 
Table 2. Summary of ESTs from adventitious roots of Liriodendron hybrids.

\begin{tabular}{l|c}
\hline & Number \\
\hline No of high quality EST sequences & 3229 \\
\hline No of contigs & 2055 \\
\hline No of singletons & 866 \\
\hline No of unigenes sequences & 2921 \\
\hline Range length of unigenes (bp) & $59-2626$ \\
\hline Average unigenes size (bp) & 1000 \\
\hline Range length of contigs (bp) & $149-2626$ \\
\hline Average contigs size (bp) & 1,010 \\
\hline Range length of singletons (bp) & $59-2349$ \\
\hline Average singletons size (bp) & 977 \\
\hline
\end{tabular}

An assembly of overlapping, contiguous sequences yielded 2921 unigenes, including 2055 contigs and 866 singletons (Table 2). The average length of the unigenes was $1000 \mathrm{bp}$ (contigs averaged $1010 \mathrm{bp}$ and singletons averaged $977 \mathrm{bp}$ ), with $59 \mathrm{bp}$ being the shortest and $2626 \mathrm{bp}$ being the longest. Of the final unigene set, 720 unigenes were 800-1000 bp and 984 unigenes were $1000 \mathrm{bp}$ or longer (Figure 2).

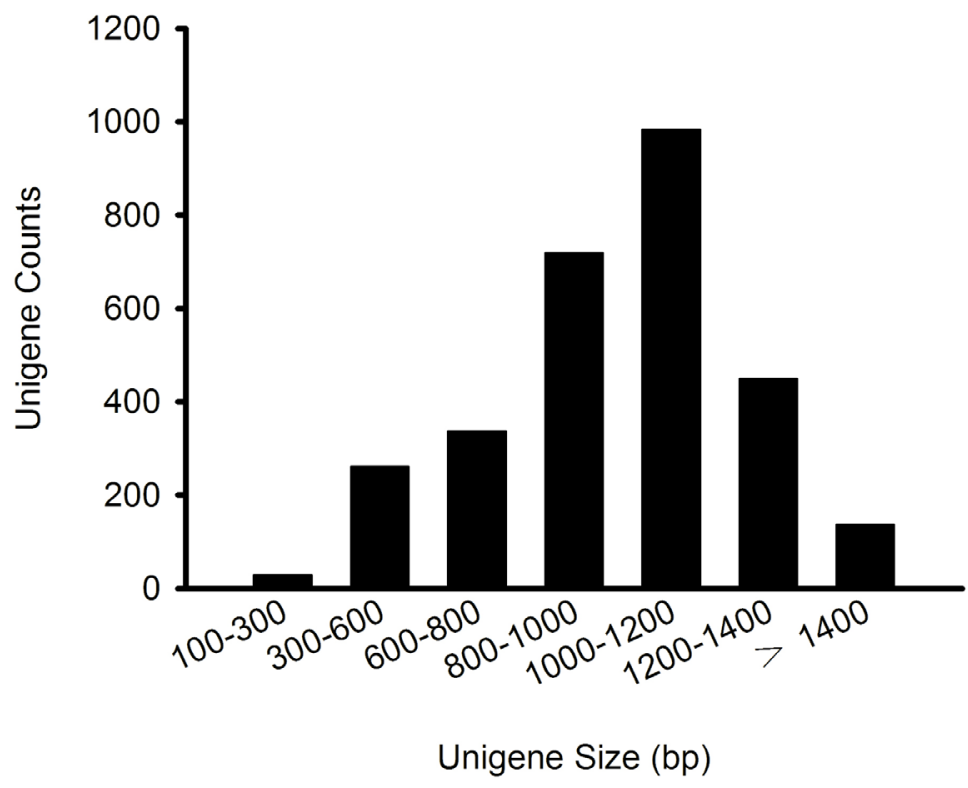

Figure 2. Distribution of unigene size.

\section{Functional classification of transcriptome}

To identify the unigenes that were potentially encoding proteins with known functions, BLASTx analysis was performed against the non-redundant protein database. A total of 2547 (87.2\%) unigenes showed significant similarity with at least one expressed, hypothetical, or unknown protein, including 1796 contigs and 751 singletons. Among those unigenes, 468 unigenes (184 singletons and 284 contigs) had high similarity with unknown proteins. However, $374(12.8 \%)$ of the unigenes did not display significant similarity to any protein 
in the database. Those unigenes could be expressed specifically during the development of adventitious roots of the Liriodendron hybrids. When compared to model species, the best BLASTX matches were with sequences from grape, poplar, rice, and Arabidopsis. Woody angiosperm species had more hits than non-woody species (Liang et al., 2011).

Detailed functional annotation of the unigenes was performed following the GO scheme. The terms were organized into three categories representing molecular functions, cellular components, and biological processes. A total of 2921 unigenes were annotated with 3760 annotations distributed in three categories (Figure 3), among which, 1341 (45.9\%) unigenes could be assigned putative molecular functions, 1196 (40.9\%) putatively contributed to cellular components, and 1,223 (41.9\%) were predicted to be involved in biological processes. A wide variety of putative functions were shown in this database. With regard to the molecular functions, classes were mainly involved in catalytic activity $(7.13 \%)$, protein binding $(5.43 \%)$, and transferase activity $(3.48 \%$; Figure $3 \mathrm{~A})$. Unigenes related to cellular components were mostly assigned to membrane $(8.01 \%)$, chloroplast $(5.80 \%)$, and cellular component (3.88\%; Figure 3B). Simultaneously, the biological process category mainly comprised of unigenes for metabolism (7.18\%), amino acid phosphorylation $(6.46 \%)$, and biosynthesis (5.35\%; Figure 3C).

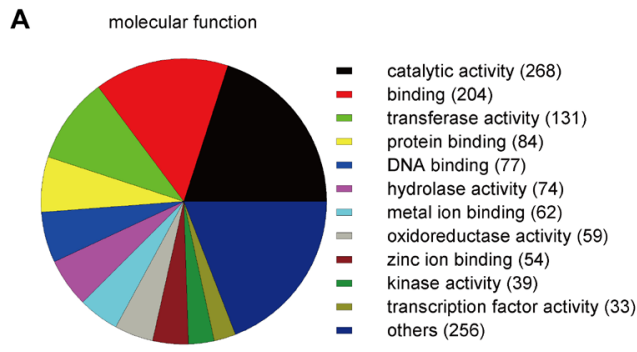

B cellular component
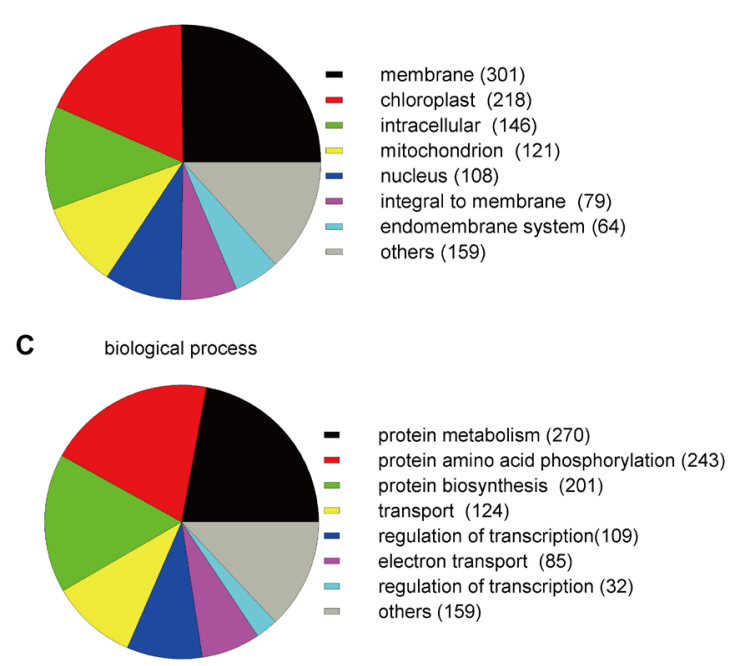

Figue 3. Pie chart representation of gene ontology (GO) annotation of EST functions in Liriodendron hybrids. A. Putative molecular functions; B. putative cellular components; C. putative biological process. 


\section{Genes for adventitious rooting}

Previous studies showed that adventitious rooting was regulated by auxin-responsive or some other genes. In the present study, many genes associated with adventitious root

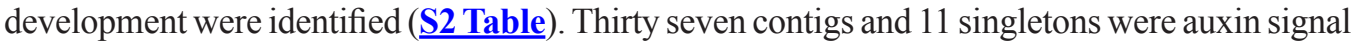
transduction-related genes induced during adventitious rooting. Aux/IAA, GH3 (Gretchen Hagen 3), and SAUR (small auxin up RNA) gene families are considered to be primary auxin-response genes (Abel and Theologis, 1996), most of which were induced immediately by auxin. AUX/IAA is a transcription suppressor and its expression level increases instantly when induced by auxin. SAUR may be involved in the auxin signal transduction pathway of calcium and calmodulin (Yang and Poovaiah, 2000). GH3 regulates the IAA level by conjugating amino acids to IAA. When IAA is added in vitro, primary root growth in the mutants, gh3.1-1, gh3.17-1, gh3.2-1, and gh3.5-1 was much slower than that in wild type Arabidopsis (Staswick et al., 2005). Furthermore, the expression of the $\mathrm{GH} 3$ gene was positively correlated with the number of adventitious roots (Sorin et al., 2006). We identified six contigs (Contig89, Contig129, Contig891, Contig524, Contig1358, and Contig1861), one contig (Contig818), and one singleton (C05-K04305603) to be highly homologous to the $A u x / I A A, G H 3$, and SAUR genes, respectively.

Additionally, eight contigs (Contig524, Contig1095, Contig1131, Contig1358, Contig1451, Contig1482, Contig1860, and Contig2053) and two singletons (C12K04301531-K1531 and E11-K04302389-K2389) were highly homologous to the $A R F$ (auxin response factor) genes. $A R F$ regulates auxin signaling by mediating the expression of auxinresponsive genes at the transcriptional level (Guilfoyle and Hagen, 2007). ARF17 is a negative regulator, whereas $A R F 6$ and $A R F 8$ are positive regulators of adventitious rooting (Gutierrez et al., 2009). They regulate the expression of the GH3.3, GH3.5, and GH3.6 genes, which are required for fine-tuning the adventitious root initiation in the Arabidopsis hypocotyl (Sorin et al., 2005; Gutierrez et al., 2012). Two contigs (Contig490 and Contig1073) and one singleton (C03-K04304147-K4147) were highly homologous to the TIRl gene. TIRl is an auxin receptor which mediates Aux/IAA degradation and finally the expression levels of the auxin-regulated genes (Dharmasiri et al., 2005). In Liriodendron hybrids, many auxin-response genes were expressed during adventitious root development, suggesting that the interaction of auxin signal transduction related genes regulated the adventitious rooting.

Both SCR (Scarecrow) and SHR (Short Root) are members of the GRAS family of computative transcription factors. The SHR-SCR signaling pathway plays a key role in stem cell maintenance and quiescent center specification in Arabidopsis (Sabatini et al., 2003). The expression of homologs of SCR and SHR genes (Contig228, Contig1131, and D05-G0907296065-996) implies that some cells may serve as stem cells in the adventitious rooting of Liriodendron hybrids. However, the existence of stem cells in hardwood cuttings is still not clear and needs further research. In Arabidopsis, NAC (NAM/ATAF/CUC) is a transcription factor, which is expressed specifically in the roots. Its expression was induced by IAA to increase the number of lateral roots (Xie et al., 2000; Guo et al., 2005). One contig and five singletons were homologous to $N A C$ in our EST database, which might have functions in the lateral root formation during adventitious rooting.

\section{Expression analysis by RT-PCR}

As shown in Figure 4, different genes were expressed constitutively at different tissues 
but had different expression patterns. For example, $N A C 1$, IRE, and $F B 1$ genes were all highly expressed in the adventitious roots. The expression of $I R E$ in the adventitious roots was more than three times higher than that in the stems and leaves. However, the expression of $A U X 1$ and $R H D 1$ genes in the adventitious roots was much lower than that of stems and leaves. ARF1 and RHL1 were more highly expressed in stems, than in adventitious roots and leaves. The expression of $A U X 1, A R F 1, N A C 1, R H D 1$, and IRE genes increased with the development of the adventitious roots, except for slight reduction in the expression of $A R F 1$ and NACl genes during the phase II and rapid reduction in the expression of the RHD1 gene during the phase III. These results suggested that the expression of auxin-related genes was induced by adventitious rooting. However, the gene expression profiles of $R H L 1$ and $F B 1$ remained nearly the same in the four phases. The increment of RHL1 and FB1 gene expression levels in phase I and phase III suggested that the induction of adventitious root primordium was regulated by the exogenous auxin and that the elongation of the adventitious roots was controlled by endogenous auxin.
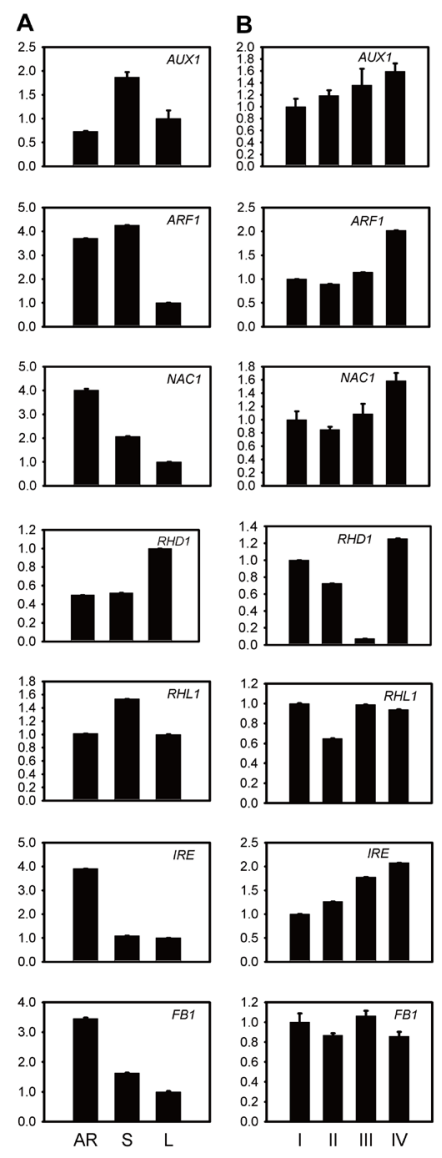

Figure 4. Expression of different genes by RT-PCR. A. Expression of seven genes in adventitious roots (AR), stems (S), and leaves (L); B. Expression of seven genes in four different developmental phases (I, II, III, IV) of adventitious roots. 
Auxin is synthesized in specific tissues and is transported by carriers. The redistribution of different auxins is carried out by different influx ( $A U X 1$ gene family) and efflux carriers (PIN gene family; Teale et al., 2006). In trees, auxin is transported actively through the vascular cambium, whereas it is actively transported through the vascular parenchyma in Arabidopsis (Marchant et al., 2002). This is further supported by the fact that AUX1 mRNA existed in stems and leaves, and accumulated rapidly with the adventitious root development. Consistent with the aux1 mutation in Arabidopsis (Bennett et al., 1996), these results revealed that auxin induced $A U X 1$ expression, and might eventually control the root formation in Liriodendron hybrids.

The ARF and AUX/IAA proteins regulate auxin-mediated transcriptional activation/ repression in Arabidopsis (Teale et al., 2006). They control adventitious root initiation by regulating $A R F$ transcripts and miRNA abundance (Gutierrez et al., 2009). In Arabidopsis, ARF1 was expressed at low levels in all the organs tested, including roots, stems, leaves, flowers, and siliques. In mango tree wood, MiARF1 was cloned from the cotyledon section during the adventitious root formation, and was expressed in both rooting and non-rooting tissues (Xiao et al., 2004). However, our results showed that $A R F 1$ was more highly expressed in stems and adventitious roots than in leaves. Moreover, in contrast to the earlier reports, its expression increased with the adventitious root formation. These results implied that ARF1 might serve different functions in the adventitious rooting in Liriodendron hybrids and other species.

$\mathrm{NACl}$ is a transcription activator in the auxin signaling pathway in Arabidopsis lateral root development. $\mathrm{NACl}$ is expressed mainly in roots, and its mRNA levels positively correlate with the lateral root number (Xie et al., 2000; Guo et al., 2005). Similar expression patterns were also found in hybrid yellow poplar, which suggested a similar function of the $N A C l$ gene in lateral root formation in adventitious rooting.

Several studies on root hair initiation had previously been reported (Seifert et al., 2002). For example, RHD1 is ubiquitously expressed in roots, leaves, stems, and flowers, and controls normal root hair initiation (Seifert et al., 2002). The rhll mutation, which led to extremely low transcript levels throughout the plant, inhibited primary root hair formation and produced a lethal seedling phenotype (Schneider et al., 1998). In Liriodendron hybrids, RHD1 and RHL1 transcripts were detected in each tissue at very low levels. Moreover, auxin induced RHDI gene expression during adventitious rooting and might control proper root hair initiation. However, there was a rapid reduction in RHDI expression in phase III of adventitious rooting, suggesting the necessity of $R H D 1$ inhibition during adventitious rooting especially during the emergence of adventitious roots from the lentice or the callus of the cuttings. IRE was strongly expressed in the elongating root hair cells, and the root hairs of the ire mutant were about 40\% shorter than the wild type in Arabidopsis (Oyama et al., 2002). In Liriodendron hybrids, IRE gene transcripts appeared to accumulate at relatively higher levels in roots than in other organs, and the expression of $I R E$ was strongly induced by auxin, suggesting its importance in root hair elongation.

We isolated the full length $F B 1$ gene in Liriodendron hybrids. The putative protein had high similarity with the F-box proteins of other species (Sun XY and Yu FX, unpublished results). The F-box families, such as TIRI and $A F B 3$ (auxin signaling F-box 3), act as auxin receptors in the auxin signaling pathway, implying their key roles in adventitious rooting. The FB1 transcript level was slightly higher in phase I and II, which suggested that FBI was involved in the early induction of adventitious root primordium by auxin and regulated the elongation of adventitious roots by endogenous auxin in the hybrid yellow poplar. The 
characteristics of their expression patterns will provide crucial information for the identification of functional mechanisms of adventitious rooting in Liriodendron hybrids and facilitate the vivid comprehension of the functional contribution of genes to metabolic pathways.

\section{SSR mining}

SSR data sets may facilitate marker development and can be applied to molecular breeding, linkage map construction, evolutionary, phylogenetic, and population genetic studies in Liriodendron hybrids (Kantety et al., 2002). In the present study, SSRs longer than $18 \mathrm{bp}$ were identified in 2,921 ESTs and 181 potential SSRs were identified in the EST data set of Liriodendron hybrids, with 166 (5.7\%) ESTs containing at least one of the considered (di-, tri-, tetra-, penta-, and hexa-) SSR motifs. This is consistent with the frequency of SSRcontaining ESTs found in L. tulipifera (5.6\%; Xu et al., 2010), but is higher than the frequency reported in rice $(4.7 \%)$, wheat $(3.2 \%)$, and maize (1.5\%; Kantety et al., 2002). This result suggests that there are abundant SSRs in the genome of hybrid yellow poplar. On an average, one SSR was found per $16.16 \mathrm{~kb}$ among the $2.92 \mathrm{Mb}$ ESTs that were sought. Among the 156 ESTs containing SSRs, 155 ESTs had one SSR and 15 ESTs had two SSRs. The frequency of SSR unit sizes was not evenly distributed (Figure 5): 58.0\% were dimers and 33.1\% were trimers. Interestingly, there were only five $(2.8 \%)$ tetramers and two $(1.1 \%)$ pentamers. Dimer repeats were the most abundant SSRs that were also observed in L. tulipifera (Liang et al., 2008; Liang et al., 2011); in contrast, trimers mainly contributed to SSR-containing ESTs in rice, soybean, and Arabidopsis (Scott et al., 2000).

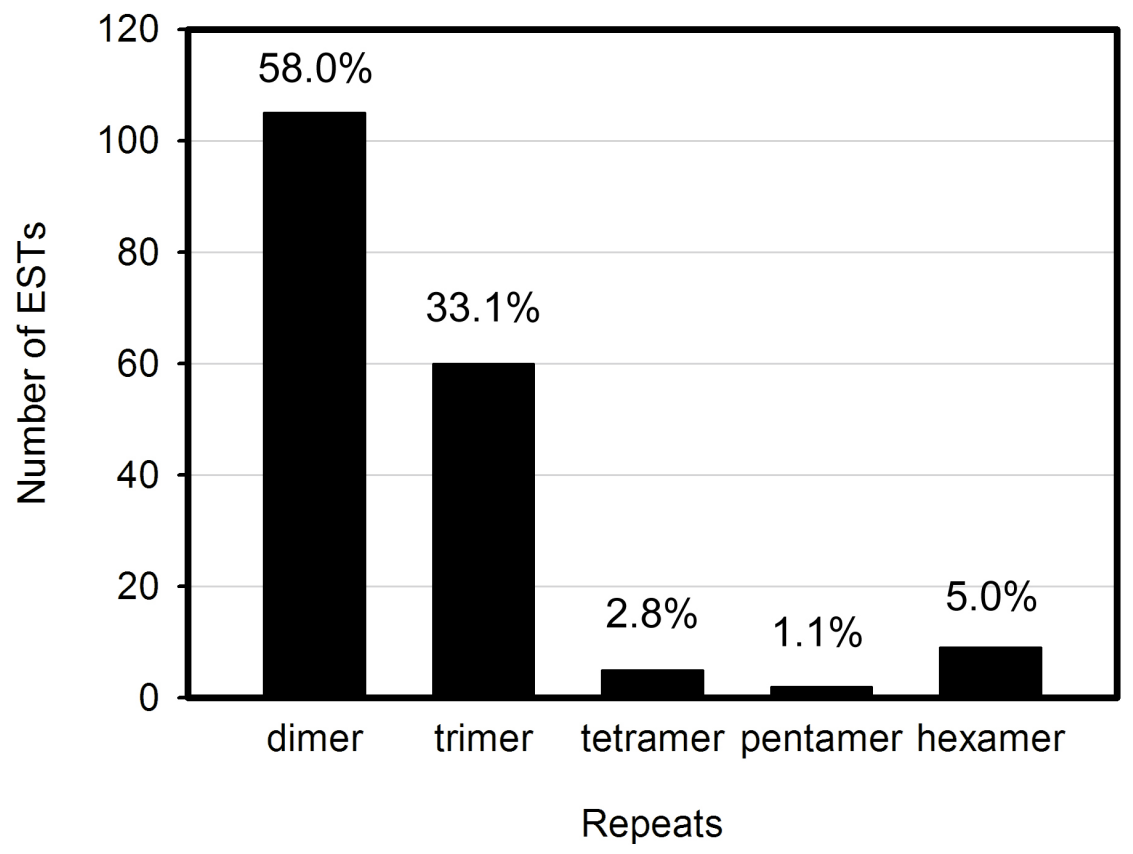

Figure 5. Frequency of SSRs in the Liriodendron hybrids unigene sets. 
Among the dimer repeats, GA/TC (56.9\%) was the most frequent dinucleotide repeat and $\mathrm{GC} / \mathrm{GC}$ was the least frequent in the Liriodendron hybrid EST dataset. Similar results had been reported in many other plant species including citrus, Arabidopsis, barley, wheat, maize, and rice ( $\mathrm{Li}$ et al., 2012). The rarity of $\mathrm{GC}$ repeats in eukaryotic genomes might be a result of the tendency of $\mathrm{CpG}$ sequences to be methylated, which could inhibit transcription (GonzalezIbeas et al., 2007). The most frequent repeats among the trimers were AAG/CTT (14.9\%), followed by GCA/TGC, and ATC/GAT (11.0 and 2.76\%), respectively, and the least frequent was ACA/TGT (Table 3). This is consistent with previous findings that indicated AAG/AGA/ GAA was the most dominant trinucleotide repeats in Arabidopsis and Rosa (Jung et al., 2005).

Table 3. Expression frequency of 2055 adventitious root contigs of Liriodendron hybrids.

\begin{tabular}{l|c|c|c}
\hline Frequency & No of contigs & No of EST & Percentage (\%) \\
\hline 2 & 1098 & 2196 & 50.95 \\
\hline 3 & 29 & 87 & 2.02 \\
\hline 4 & 9 & 36 & 0.84 \\
\hline $5-16$ & 13 & 109 & 2.53 \\
\hline $24-48$ & 6 & 231 & 5.36 \\
\hline $50-119$ & 4 & 318 & 7.38 \\
\hline 130 & 1 & 130 & 3.02 \\
\hline 173 & 1 & 173 & 4.01 \\
\hline 204 & 1 & 204 & 4.73 \\
\hline 250 & 1 & 250 & 5.80 \\
\hline 284 & 1 & 284 & 6.59 \\
\hline
\end{tabular}

One hundred and twenty-two ESTs containing 127 SSRs were selected for SSR primer modeling and for primer generation (3 Table). Among those primers, $115(90.6 \%)$ produced clear bands in Liriodendron hybrids, L. chinense, and L. tulipifera. Sixty one (48.0\%) ESTSSR markers were found to be polymorphic in eight Liriodendron hybrids, suggesting a high level of cross-species transferability. This is in agreement with the report that the L. tulipifera SSRs may be similarly vital in related species of the same family (Xu et al., 2010).

To date, there has been no EST library for Liriodendron hybrids. Only a few miRNAs have been reported during somatic embryogenesis. The present research generated and characterized a normalized uncut cDNA library for Liriodendron hybrid adventitious roots, identified 5176 ESTs, and then obtained 2921 unigenes (2,055 contigs and 866 singletons, ranging from 59 to $2626 \mathrm{bp}$ ). Most of the unigenes (87.2\%) had similarity to the publicly available plant protein sequences, and 2921 unigenes were annotated with 3760 annotations distributed among the three categories using the GO scheme, representing a wide variety of putative functions. Many genes might play important roles in the development of adventitious roots in Liriodendron hybrids, such as genes related to auxin signal transduction, transcription factors, root meristem maintenance, and root hair development. Furthermore, the spatial expression patterns indicated that $A U X 1, I R E$, and $F B 1$ were highly expressed in the adventitious roots, and that the expression of $A U X 1, A R F 1, N A C 1, R H D 1$, and IRE was induced during the adventitious rooting, suggesting the potential functions of related genes during adventitious rooting of hybrid yellow poplar. In addition, 181 SSRs were identified based on EST sequences and 115 EST-SSR markers produced clear bands in the genus Liriodendron, implying that these markers could be applied in genetic mapping and genetic diversity among Liriodendron. This study is the first to identify genes associated with adventitious rooting in Liriodendron and provides numerous cues for future genomic studies on Liriodendron hybrids. 


\section{Conflicts of interest}

The authors declare no conflict of interest.

\section{ACKNOWLEDGMENTS}

We thank Professor Li'an Xu for providing advice on experimental design and thank Professor Huixin Pan for providing partial experiment material of Liriodendron hybrids. We are also grateful to Cong Jiang and Tengyun Liu for technical support and to Shujuan Liu, Sheng Zhu, and Wei Zhu for their help with bioinformatics analysis. We also express appreciation to Zhenbang Wei (Invitrogen, Shanghai, China) for assisting with the normalized cDNA library. Research supported by the National Natural Science Foundation of China (\#381272) and the Training Plan of Academic Leaders Program of Jiangxi Province (\#20133BCB22012).

\section{REFERENCES}

Abel S and Theologis A (1996). Early genes and auxin action. Plant Physiol. 111: 9-17. http://dx.doi.org/10.1104/ pp.111.1.9

Albert VA, Soltis DE, Carlson JE, Farmerie WG, et al. (2005). Floral gene resources from basal angiosperms for comparative genomics research. BMC Plant Biol. 5: 5. http://dx.doi.org/10.1186/1471-2229-5-5

Bennett MJ, Marchant A, Green HG, May ST, et al. (1996). Arabidopsis AUX1 gene: a permease-like regulator of root gravitropism. Science 273: 948-950. http://dx.doi.org/10.1126/science.273.5277.948

Dharmasiri N, Dharmasiri S and Estelle M (2005). The F-box protein TIR1 is an auxin receptor. Nature 435: 441-445. http://dx.doi.org/10.1038/nature03543

Gonzalez-Ibeas D, Blanca J, Roig C, González-To M, et al. (2007). MELOGEN: an EST database for melon functional genomics. BMC Genomics 8: 306. http://dx.doi.org/10.1186/1471-2164-8-306

Guilfoyle TJ and Hagen G (2007). Auxin response factors. Curr. Opin. Plant Biol. 10: 453-460. http://dx.doi.org/10.1016/j. pbi.2007.08.014

Guo HS, Xie Q, Fei JF and Chua NH (2005). MicroRNA directs mRNA cleavage of the transcription factor NACl to downregulate auxin signals for arabidopsis lateral root development. Plant Cell 17: 1376-1386. http://dx.doi. org $/ 10.1105 /$ tpc. 105.030841

Gutierrez L, Bussell JD, Pacurar DI, Schwambach J, et al. (2009). Phenotypic plasticity of adventitious rooting in Arabidopsis is controlled by complex regulation of AUXIN RESPONSE FACTOR transcripts and microRNA abundance. Plant Cell 21: 3119-3132. http://dx.doi.org/10.1105/tpc.108.064758

Gutierrez L, Mongelard G, Floková K, Pacurar DI, et al. (2012). Auxin controls Arabidopsis adventitious root initiation by regulating jasmonic acid homeostasis. Plant Cell 24: 2515-2527. http://dx.doi.org/10.1105/tpc.112.099119

Jin H, Do J, Moon D, Noh EW, et al. (2011). EST analysis of functional genes associated with cell wall biosynthesis and modification in the secondary xylem of the yellow poplar (Liriodendron tulipifera) stem during early stage of tension wood formation. Planta 234: 959-977. http://dx.doi.org/10.1007/s00425-011-1449-1

Jung S, Abbott A, Jesudurai C, Tomkins J, et al. (2005). Frequency, type, distribution and annotation of simple sequence repeats in Rosaceae ESTs. Funct. Integr. Genomics 5: 136-143. http://dx.doi.org/10.1007/s10142-005-0139-0

Kantety RV, La Rota M, Matthews DE and Sorrells ME (2002). Data mining for simple sequence repeats in expressed sequence tags from barley, maize, rice, sorghum and wheat. Plant Mol. Biol. 48: 501-510. http://dx.doi. org/10.1023/A:1014875206165

Kuhl JC, Cheung F, Yuan Q, Martin W, et al. (2004). A unique set of 11,008 onion expressed sequence tags reveals expressed sequence and genomic differences between the monocot orders Asparagales and Poales. Plant Cell 16: 114-125. http://dx.doi.org/10.1105/tpc.017202

Li T, Chen J, Qiu S, Zhang Y, et al. (2012). Deep sequencing and microarray hybridization identify conserved and speciesspecific microRNAs during somatic embryogenesis in hybrid yellow poplar. PLoS One 7: e43451. http://dx.doi. org/10.1371/journal.pone.0043451

Li SW, Xue L, Xu S, Feng H, et al. (2009). Mediators, Genes and Signaling in Adventitious Rooting. Bot. Rev. 75: 230247. http://dx.doi.org/10.1007/s12229-009-9029-9 
Liang H, Ayyampalayam S, Wickett N, Barakat A, et al. (2011). Generation of a large-scale genomic resource for functional and comparative genomics in Liriodendron tulipifera L. Tree Genet. Genomes 7: 941-954. http://dx.doi. org/10.1007/s11295-011-0386-2

Liang H, Carlson JE, Leebens-Mack JH, Wall PK, et al. (2008). An EST database for Liriodendron tulipifera L. floral buds: the first EST resource for functional and comparative genomics in Liriodendron. Tree Genet. Genomes 4: 419433. http://dx.doi.org/10.1007/s11295-007-0120-2

Liang H, Fang EG, Tomkins JP, Luo M, et al. (2007). Development of a BAC library for yellow-poplar (Liriodendron tulipifera) and the identification of genes associated with flower development and lignin biosynthesis. Tree Genet. Genomes 3: 215-225. http://dx.doi.org/10.1007/s11295-006-0057-x

Marchant A, Bhalerao R, Casimiro I, Eklöf J, et al. (2002). AUX1 promotes lateral root formation by facilitating indole3-acetic acid distribution between sink and source tissues in the Arabidopsis seedling. Plant Cell 14: 589-597. http:// dx.doi.org/10.1105/tpc.010354

Navarro L, Dunoyer P, Jay F, Arnold B, et al. (2006). A plant miRNA contributes to antibacterial resistance by repressing auxin signaling. Science 312: 436-439. http://dx.doi.org/10.1126/science.1126088

Oyama T, Shimura Y and Okada K (2002). The IRE gene encodes a protein kinase homologue and modulates root hair growth in Arabidopsis. Plant J. 30: 289-299. http://dx.doi.org/10.1046/j.1365-313X.2002.01290.x

Parks CR, Miler NG, Wendel JF and Mcdouglas KM (1983). Genetic divergence within the genus Liriodendron (Magnoliaceae). Ann. Mo. Bot. Gard. 70: 658-666. http://dx.doi.org/10.2307/2398983

Parks CR and Wendel JF (1990). Molecular divergence between Asian and North American species of Liriodendron (Magnoliaceae) with implications for interpretation of fossil floras. Am. J. Bot. 77: 1243-1256. http://dx.doi. org/10.2307/2444585

Ramírez-Carvajal GA, Morse AM, Dervinis C and Davis JM (2009). The cytokinin type-B response regulator PtRR13 is a negative regulator of adventitious root development in Populus. Plant Physiol. 150: 759-771. http://dx.doi. org/10.1104/pp.109.137505

Sabatini S, Heidstra R, Wildwater M and Scheres B (2003). SCARECROW is involved in positioning the stem cell niche in the Arabidopsis root meristem. Genes Dev. 17: 354-358. http://dx.doi.org/10.1101/gad.252503

Schneider K, Mathur J, Boudonck K, Wells B, et al. (1998). The ROOT HAIRLESS 1 gene encodes a nuclear protein required for root hair initiation in Arabidopsis. Genes Dev. 12: 2013-2021. http://dx.doi.org/10.1101/gad.12.13.2013

Scott KD, Eggler P, Seaton G, Rossetto M, et al. (2000). Analysis of SSRs derived from grape ESTs. Theor. Appl. Genet. 100: 723-726. http://dx.doi.org/10.1007/s001220051344

Seifert GJ, Barber C, Wells B, Dolan L, et al. (2002). Galactose biosynthesis in Arabidopsis: genetic evidence for substrate channeling from UDP-D-galactose into cell wall polymers. Curr. Biol. 12: 1840-1845. http://dx.doi.org/10.1016/ $\underline{\mathrm{S} 0960-9822(02) 01260-5}$

Shimizu-Sato S, Tanaka M and Mori H (2009). Auxin-cytokinin interactions in the control of shoot branching. Plant Mol. Biol. 69: 429-435. http://dx.doi.org/10.1007/s11103-008-9416-3

Sorin C, Bussell JD, Camus I, Ljung K, et al. (2005). Auxin and light control of adventitious rooting in Arabidopsis require ARGONAUTE1. Plant Cell 17: 1343-1359. http://dx.doi.org/10.1105/tpc.105.031625

Sorin C, Negroni L, Balliau T, Corti H, et al. (2006). Proteomic analysis of different mutant genotypes of Arabidopsis led to the identification of 11 proteins correlating with adventitious root development. Plant Physiol. 140: 349-364. http://dx.doi.org/10.1104/pp.105.067868

Staswick PE, Serban B, Rowe M, Tiryaki I, et al. (2005). Characterization of an Arabidopsis enzyme family that conjugates amino acids to indole-3-acetic acid. Plant Cell 17: 616-627. http://dx.doi.org/10.1105/tpc.104.026690

Teale WD, Paponov IA and Palme K (2006). Auxin in action: signalling, transport and the control of plant growth and development. Nat. Rev. Mol. Cell Biol. 7: 847-859. http://dx.doi.org/10.1038/nrm2020

Xiao JN, Huang XL, Huang X and Li XJ (2004). [Identification of an auxin response factor-like protein cDNA from mango cotyledon section]. Sheng Wu Gong Cheng Xue Bao 20: 59-62.

Xie Q, Frugis G, Colgan D and Chua NH (2000). Arabidopsis NAC1 transduces auxin signal downstream of TIR1 to promote lateral root development. Genes Dev. 14: 3024-3036. http://dx.doi.org/10.1101/gad.852200

Xu M, Sun YG and Li HG (2010). EST-SSRs development and paternity analysis for Liriodendron spp. New For. 40: 361-382. http://dx.doi.org/10.1007/s11056-010-9205-0

Yang T and Poovaiah BW (2000). Molecular and biochemical evidence for the involvement of calcium/calmodulin in auxin action. J. Biol. Chem. 275: 3137-3143. http://dx.doi.org/10.1074/jbc.275.5.3137

Yang Y, Xu M, Luo Q, Wang J, et al. (2014). De novo transcriptome analysis of Liriodendron chinense petals and leaves by Illumina sequencing. Gene 534: 155-162. http://dx.doi.org/10.1016/j.gene.2013.10.073

Zhang XP and Fang YM (2003). Anatomical observation of the origin and development of adventitious roots in hybrid tuliptrees during cutting. J. Plant Res. Environment. 12: 10-15. 


\section{Supplementary material}

Table S1. CT values of RT-PCR of housekeeping genes.

Table S2. Genes associated with adventitious roots development in Liriodendron hybrids.

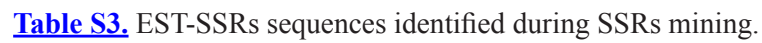

